\title{
Neural Mass Model-Based Different EEG Signal Generation and Analysis in Simulink
}

\author{
Sheikh Md. Rabiul Islam, Md. Shakibul Islam
}

\begin{abstract}
The electroencephalogram (EEG) is an electrophysiological monitoring strategy that records the spontaneous electrical movement of the brain coming about from ionic current inside the neurons of the brain. The importance of the EEG signal is mainly the diagnosis of different mental and brain neurodegenerative diseases and different abnormalities like seizure disorder, encephalopathy, dementia, memory problem, sleep disorder, stroke, etc. The EEG signal is very useful for someone in case of a coma to determine the level of brain activity. So, it is very important to study EEG generation and analysis. To reduce the complexity of understanding the pathophysiological mechanism of EEG signal generation and their changes, different simulation-based EEG modeling has been developed which are based on anatomical equivalent data. In this paper, Instead of a detailed model a neural mass model has been used to implement different simulation-based EEG models for EEG signal generation which refers to the simplified and straightforward method. This paper aims to introduce obtained EEG signals of own implementation of the Lopes da Silva model, Jansen-Rit model, and Wendling model in Simulink and to compare characteristic features with real EEG signals and better understanding the EEG abnormalities especially the seizure-like signal pattern.
\end{abstract}

Keywords: EEG signal, EEG abnormalities, Neural Mass Model, Simulink.

\section{INTRODUCTION}

$\mathrm{T}$

The electroencephalogram (EEG) is a proficient methodology of recording the spontaneous electrical action of the brain as recorded from numerous electrodes put on the scalp [1] An Evoked potential records the electric potential produced from stimulation of the sensory pathway. The extracellular current flow inside the neurons of the brain related to the summed postsynaptic potential of synchronously enacted and vertically oriented neurons come about in the EEG and EP [2].

Neurophysiology enthusiasts have a great interest in the nervous system information processing and behavior controlling technique. EEG signal modeling, an analytical

Manuscript received on 24 June 2021 | Revised Manuscript received on 30 June 2021 | Manuscript Accepted on 15 August 2021 | Manuscript published on 30 August 2021.

* Correspondence Author

Sheikh Md. Rabiul Islam*, Department of Electronics and Communication Engineering, Khulna University of Engineering \& Technology, Khulna, Bangladesh. Email: robi@ece.kuet.ac.bd

Md. Shakibul Islam, Department of Electronics and Communication Engineering, Khulna University of Engineering \& Technology, Khulna, Bangladesh. Email: islam1609027@stud.kuet.ac.bd

(C) The Authors. Published by Lattice Science Publication (LSP). This is an open access article under the CC-BY-NC-ND license (http://creativecommons.org/licenses/by-nc-nd/4.0/) approach that includes functional properties of neurons is utilized to distinguish pathophysiological EEG changes that are vital in clinical diagnosis. Simulation-based EEG signal modeling is important for a better understanding of the physical mechanism of EEG signal generation, EEG abnormalities which can be accomplished by analyzing the estimated model parameters, anticipating long haul neurological outcome, and illustrate the efficacy of a strategy of certain quantitative analysis [3]. To simulate electrophysiological brain activity, different numerical models have been developed such as the Lopes da Silva model, Jensen-Rit model, Wendling model. The mathematical models are developed by simple circuits rather than complex cortical connections and the model parameters are based on anatomical data, such as the number of neural connections or the excitability of neurons. Then the models can be utilized to test their particular roles and impact [2].

There are different sorts of EEG signals like alpha, beta, and gamma, delta, theta based on signal frequencies which ranges from $0.1 \mathrm{~Hz}$ to more than $100 \mathrm{~Hz}$. In this paper mainly three macroscopic mathematical models which are called neural mass models have been discussed to generate three types of signal (Alpha, Beta, and Gamma). Within the neural mass model, neuronal systems of the cortex are considered as a spatially persistent network and the dynamics of the cortical locale summarized into few state variables that represent the mean firing rate of the cell(Soma), which imitate the interaction of excitatory and inhibitory neurons populations, organized in an input loop [4] [5]. Particularly, applying the neural mass model, simulation of alpha rhythm activity were performed by Freeman's model [6], Lopez da Silva's model [7], in the late seventies, and then improved and extended by Jansen and Rit [2] to simulate alpha and beta activity and Wendling et al. [8] for high-frequency gamma-band rhythms and seizure-like signal.

The inherent limitation of the neural mass model is that (i) Though the gamma rhythms can be generated by a chain of fast inhibitory interneuron without the contribution of other neurons which has been observed in recent considers, In neural mass model Gamma rhythms are generated by the interaction between pyramidal neurons and fast inhibitory interneurons where little values of the synaptic time constant are used (ii) Numerous rhythms inside the same cortical locale particularly the beta and gamma rhythms can hardly be obtained [5].

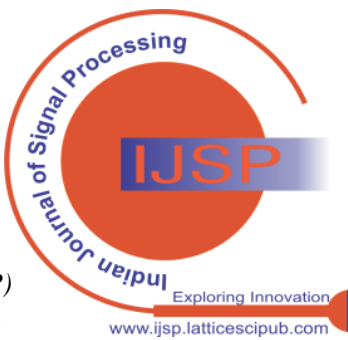


In this paper, different models for simulating different aspects of EEG signal following the real functioning of the brain have been discussed, then introduced different EEG and seizure-like signal of own implementation in Simulink by setting different parameters analogous to biological data, and finally compared those signals with real EEG signals.

\section{RELETED WORK}

In recent years many researchers give their concentration to the simulation-based EEG signal modeling for a better understanding of the electrophysiological mechanism of the EEG signal generation and the abnormality detection in the different EEG signals. In different research, there comes different models for EEG simulation. One is the Microscopic Model or detailed model [4] where the function of the brain has been described by the interaction of both the central and peripheral nervous system. The behavior of thousands of individual neurons, their connection, and relationship among those is necessary to model the potentials of the local field. Then the physiological phenomena have been represented by mathematical and computational equations. But nowadays, research interest goes to the simplified model instead of the microscopic model or detailed model because it is troublesome to find out the impact of each parameter. The second one is the macroscopic model, which is best known as the neural mass model [6], [7], [9] where the neural activity in a cortical region is summarized with simplifying assumption and empirical priors to mimic EEG signals which is same as the real EEG signals. In this approach, a linear function at the average soma and a nonlinear function at the average dendrite and white noise as the influence of all far neurons is used [4]. These models compromise macro-columns or simplified to a cortical region are summarized to one or two-state variables to speak to the average activity (mean firing rate) of the full population, for this reason, it is alluded to as a "mean-field approximation" which is proficient to determine the steady-state behavior of the neuronal framework rather than the dynamic or non-stationary context as it's utility is not established yet [10]. In most cases, the neural mass model is utilized to simulate alpha rhythms by Freemen [6], Lopes Da Silva [7], and Jansen \& Rit [2]. Following the recent studies that the kinetics of inhibitory interneurons in hippocampal have a key impact on the signals generation, the gamma-like signal has been generated [8]. To generate a realistic EEG signal, it is very important to set the parameter values analogous to the pathological data. Then the procedure of generating several EEG signals can be analyzed by different bifurcation analyses mainly the Hopf bifurcation and saddle-node bifurcation phenomenon [11], [12]. To compare the simulated EEG signal with real EEG signal the multi-frequency analysis [13] and spectral analysis [5], [8], [10] have been performed.

\section{METHODOLOGY}

The section has been discussed in different sub-section. The flow diagram of this work has been shown below in Fig. 1.

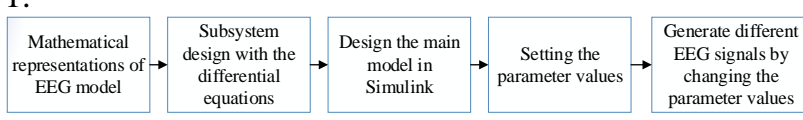

Fig. 1.Flow diagram of this work

\section{A. Lopes da Silva Model}

Lopes da Silva model has been designed for generating Alpha rhythms assuming two types of neuron (Thalamo-cortical relay Cells or TCR cells and The interneurons or IN) based on the historical data from the Ventro-posterolateral nucleus of the thalamus[14].

1) Mathematical Representation

The neural mass model has been used to design this where two conversion operations [9], one is a wave-to-pulse operator at the soma of the neuron and another a pulse-to-wave operator. The first one is a static sigmoid function which relates to the mean firing rate and the second one depends on Synaptic kinetics which represents the average postsynaptic response. [10]

The linear pulse-to-wave converter is,

$$
\begin{gathered}
h_{i}(t)=B\left[e^{-b_{1} t}-e^{-b_{2} t}\right] . \\
h_{e}(t)=B\left[e^{-a_{1} t}-e^{-a_{2} t}\right] .
\end{gathered}
$$

Here, $h_{e}(t)$ and $h_{i}(t)$ are the impulse responses for the excitatory and inhibitory synapses.[7]

The equivalent differential equations for (1) and (2) are, $\ddot{y}(t)+\left(b_{1}+b_{2}\right) y^{\circ}(t)+b_{1} b_{2} y(t)=B\left(b_{2}-b_{1} x(t)\right)$

$\ddot{y}(t)+\left(a_{1}+a_{2}\right) \dot{y}(t)+a_{1} a_{2} y(t)=A\left(a_{2}-a_{1} x(t)\right)$

And there has also a sigmoid function.

2) Setting the parameter values

Table- I: Different Parameter for Simulating Lopes da Silva Model [7]

\begin{tabular}{|l|l|l|}
\hline \multicolumn{1}{|c|}{ Parameter } & \multicolumn{1}{|c|}{ Interpretation } & \multicolumn{1}{c|}{ Standard value } \\
\hline $\mathrm{a}_{1}$ & \multirow{2}{*}{ Average time constants } & $55 \mathrm{~s}^{-1}$ \\
$\mathrm{a}_{2}$ & & $60.5 \mathrm{~s}^{-1}$ \\
$\mathrm{~b}_{1}$ & & $27.5 \mathrm{~s}^{-1}$ \\
\cline { 3 - 3 } $\mathrm{b}_{2}$ & & $5 \mathrm{~s}^{-1}$ \\
\hline $\mathrm{A}$ & Exitatory Synaptic Potential & $1.65 \mathrm{mV}$ \\
\hline $\mathrm{B}$ & Inhibitory Synaptic Potential & $32 \mathrm{mV}$ \\
\hline $\mathrm{C}_{1}$ & Number of TCR cells & 32 \\
\hline $\mathrm{C}_{2}$ & Number of IN & 3 \\
\hline $\mathrm{K}$ & Stability limit of the network & 3500000000 \\
\hline
\end{tabular}

\section{3) Designing the model}

The subsystem is designed for simplification of the main model design. The linear pulse-to-wave converter equivalent differential equation which is stated in (3) has been designed in this section. The design of (4) is similar to the design of (3) except for the parameter values only. The designed subsystem for (3) is shown below in Fig. 2

\section{B. Jansen-Rit Model}

Jansen-Rit model is based on Lopes da Silva's model [7] which contains two kinds of neurons populations, total three interconnected neural population which are mainly involving in the generation of paroxysmal activity to generate Alpha and beta activity. The first one is Pyramidal projection neurons and the second one is interneurons (excitatory and inhibitory interneurons).

\section{Published By:}


The pyramidal projection neurons receive feedback from excitatory and inhibitory interneurons and an excitatory input from neighboring cortical units that describes the average density of afferent action potentials [13].

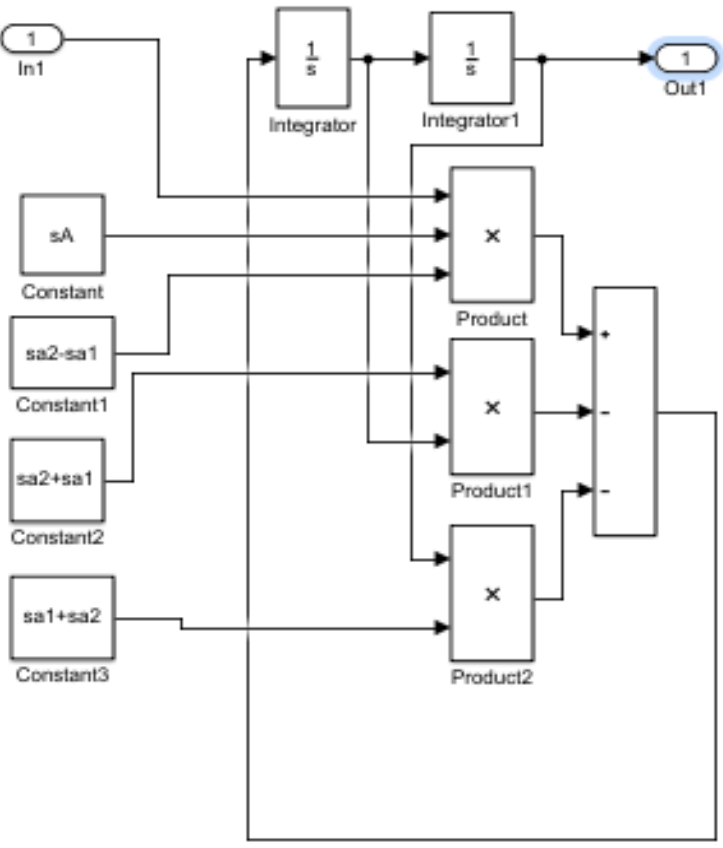

Fig. 2.Figure for designing the differential equation for pulse-to-wave converter operation.

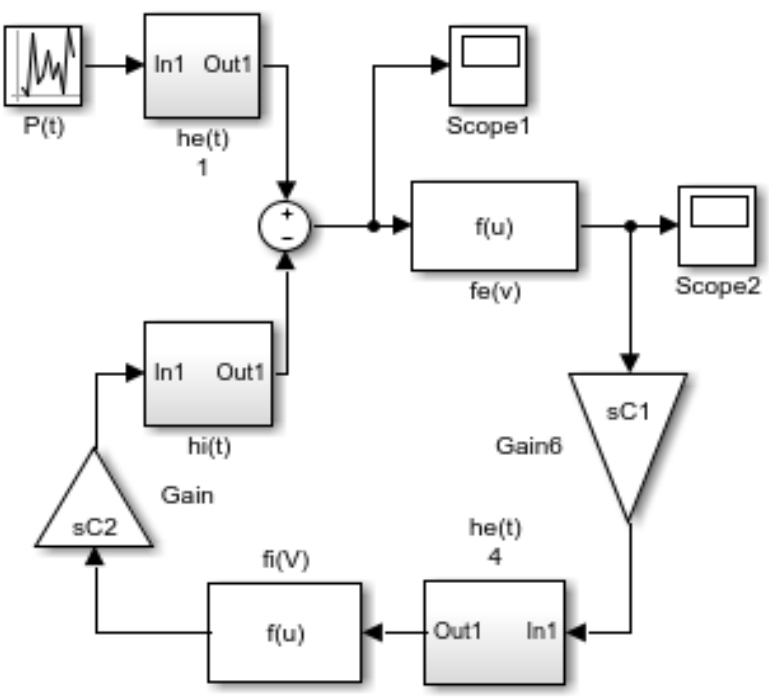

Fig. 3.Figure for designing Lopes Da Silva model in Simulink.

\section{1) Mathematical Representation}

Following the neural mass model, two conversion operator has been used to design this model like Lopes da Silva model. A pulse-to-wave converter converts the input average pulse density into an average postsynaptic membrane potential of action potentials and a wave-to-pulse converter converts the average membrane potential to the average pulse density of action potentials.

A liner impulse response is applied as a pulse-to-wave converter shown in (5) and (6). For the excitatory case the impulse response $h_{e}(t)$ is,

$$
h_{e}(t)= \begin{cases}\text { Aate } e^{-a t} & t \geq 0 \\ 0 & t<0\end{cases}
$$

For inhibitory case $h_{i}(t)$ is,

$$
h_{i}(t)= \begin{cases}B b t e^{-b t} & t \geq 0 \\ 0 & t<0\end{cases}
$$

This can be represented as a differential equation,

$$
\ddot{y}(t)=\operatorname{Aax}(t)-2 a y^{\prime}(t)-a^{2} y(t)
$$

Equation (7) can be rewritten for design consideration as $1^{\text {st }}$ order differential equations,

$$
\left\{\begin{array}{c}
\dot{y}(t)=z(t) \\
\dot{z}(t)=\operatorname{Aax}(t)-2 a z(t)-a^{2} y(t)
\end{array}\right.
$$

Where $x(t)$ is input, $y(t)$ is output.

A sigmoid function is used as a wave-to-pulse converter as shown in (9)

$$
\operatorname{Sigm}(v)=\frac{2 e_{0}}{1+e^{n\left(v_{0}-v\right)}}
$$

Where ${ }^{\nu_{0}}$ is the potential value for $50 \%$ firing rate and ${ }^{F}$ is the slope of the sigmoid function.

This model can be described through these three second-order differential equations or a set of six first-order differential equations which stated below,

\begin{tabular}{|c|c|c|}
\hline Parameter & Interpretation & Standard value \\
\hline A & Average excitatory synaptic gain & $3.25 \mathrm{mV}$ \\
\hline $\mathrm{B}$ & Average inhibitory synaptic gain & $22 \mathrm{mV}$ \\
\hline $\mathrm{a}$ & $\begin{array}{l}\text { The inverse of the Dendritic } \\
\text { average time constant in the } \\
\text { feedback excitatory loop }\end{array}$ & $100 \mathrm{~s}^{-1}$ \\
\hline $\mathrm{b}$ & $\begin{array}{l}\text { Inverse of Dendritic average time } \\
\text { constant in the feedback } \\
\text { inhibitory loop }\end{array}$ & $50 \mathrm{~s}^{-1}$ \\
\hline $\mathrm{C}_{1}$ & \multirow{2}{*}{$\begin{array}{l}\text { Average number of synaptic } \\
\text { contacts } \\
\text { in the excitatory feedback loop }\end{array}$} & $\begin{array}{l}135 \text { (For Alpha) } \\
1350 \text { (For Beta) }\end{array}$ \\
\hline $\mathrm{C}_{2}$ & & $0.8^{*} \mathrm{C}_{1}$ \\
\hline $\mathrm{C}_{3}$ & \multirow{2}{*}{$\begin{array}{l}\text { Average number of synaptic } \\
\text { contacts } \\
\text { in the slow feedback inhibitory } \\
\text { loop }\end{array}$} & $0.25^{*} \mathrm{C}_{1}$ \\
\hline $\mathrm{C}_{4}$ & & $0.25^{*} \mathrm{C}_{1}$ \\
\hline
\end{tabular}

$$
\begin{aligned}
& \dot{y}_{0}(t)=y_{3}(t) \\
& \dot{y}_{3}(t)=A a \operatorname{Sigm}\left[y_{1}(t)-y_{2}(t)\right] \\
& -2 a y_{3}(t)-a^{2} y_{0}(t) \\
& \dot{y}_{1}(t)=y_{4}(t) \\
& \dot{y}_{4}(t)=A a\left\{p(t)+C_{2} \operatorname{Sigm}\left[C_{1} y_{0}(t)\right]\right\} \\
& \dot{y}_{2}(t)=y_{5}(t) \\
& -2 a y_{4}(t)-a^{2} y_{1}(t) \\
& \tilde{y}_{5}(t)=B b\left\{C_{4} \operatorname{Sigm}\left[C_{9} Y_{0}(t)\right]\right\} \\
& -2 b y_{5}(t)-b^{2} y_{2}(t)
\end{aligned}
$$

2) Setting the parameter values

Table- II: Different Parameter for Simulating Jansen-Rit mode [2]

\section{Published By:}

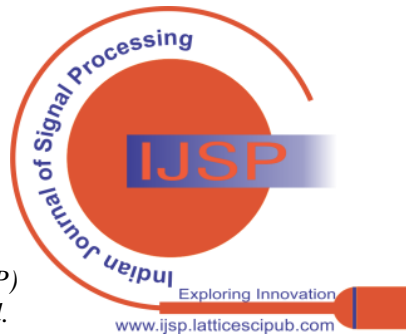


3) Designing the model

To design this model clearly and increase the readability, two subsystems have been designed for the linear impulse response $h_{e}(t)$ and $h_{i}(t)$ shown in Fig. 4. The differential equation (8) is used to design this subsystem. Design for (5) and (6) are the same except for the parameter values only. So here one design has been represented.

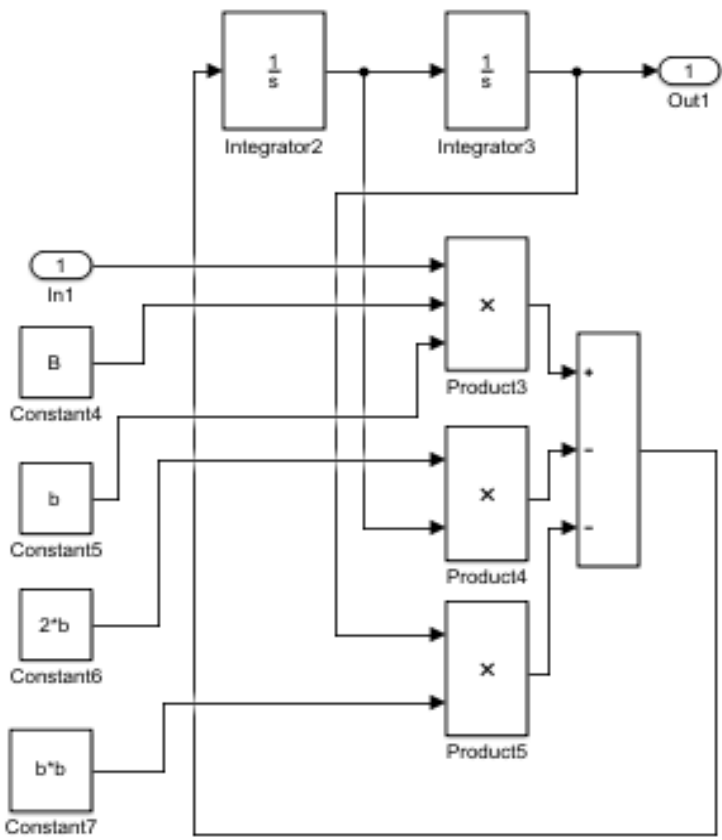

Fig. 4.Figure for designing the differential equation for pulse-to-wave converter operation.

The Jansen-Rit model has been designed to generate Alpha and Beta rhythm in Simulink using different functional blocks which are shown in Fig. 5.

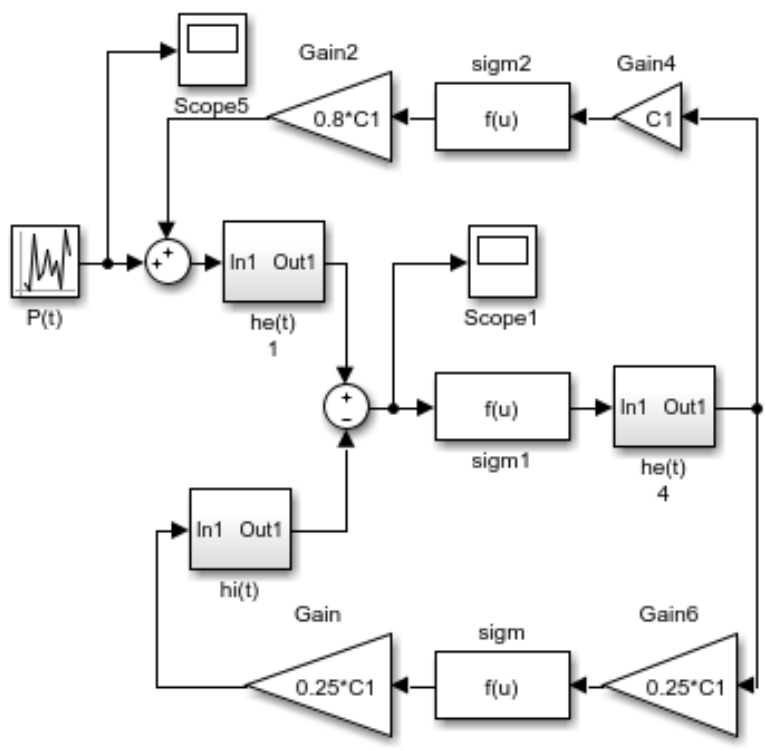

Fig. 5.Figure for designing Jansen-Rit model in Simulink.

\section{Wendling Model}

This is very much similar to the Jansen-Rit model where only a new inhibitory feedback loop is added. This model is used to generate Gamma signal and seizure-like signal as it has a subset of interneurons providing somatic inhibition to pyramidal cells which is absent in the Jansen-Rit model[8].

\section{1) Mathematical Representation}

The This model is very similar to the Jansen-Rit model. Like the previous two models, here also have a pulse-to-wave converter that converts the input average presynaptic pulse density into an average postsynaptic membrane potential of action potentials with respective impulse response [8],

For excitatory,

$$
h_{e}(t)= \begin{cases}\text { Aat } e^{-a t} & t \geq 0 \\ 0 & t<0\end{cases}
$$

For slow inhibitory,

$$
h_{i}(t)= \begin{cases}B b t e^{-b t} & t \geq 0 \\ 0 & t<0\end{cases}
$$

For fast inhibitory,

$$
h_{g}(t)= \begin{cases}G_{g t e^{-g t}} & t \geq 0 \\ 0 & t<0\end{cases}
$$

Each linear transfer function $h_{e}(t), h_{i}(t)$, and $h_{g}(t)$ introduces a pair of $1^{\text {st }}$ order ordinary differential equations shown below,

$$
\left\{\begin{array}{c}
\tilde{z}_{1}(t)=\mathbb{z}_{2}(t) \\
\tilde{\Xi}_{2}(t)=W g x(t)-2 w z_{2}(t)-w^{2} \mathbb{Z}_{1}(t)
\end{array}\right.
$$

Where $\mathrm{W}=\mathrm{A}, \mathrm{W}=\mathrm{B}$ or $\mathrm{W}=\mathrm{G}$ and $\mathrm{w}=\mathrm{a}, \mathrm{w}=\mathrm{b}$ or $\mathrm{w}=\mathrm{g}$, depending on the excitatory, slow inhibitory or fast inhibitory case. Also, $x(t)$ is the input, and $z_{1}(t)$ is the output of the linear transfer functions.

A wave-to-pulse converter is an asymmetric sigmoid function that converts the average membrane potential to the average pulse density of action potentials.

$$
\operatorname{Sigm}(v)=\frac{2 e_{0}}{1+e^{\text {n( }\left(\mathrm{v}_{0}-\mathrm{v}\right)}}
$$

Where ${ }^{\nu_{0}}$ is the potential value for $50 \%$ firing rate and ${ }^{r}$ is the slope of the sigmoid function

The connectivity constants $C_{1}$ to $C_{7}$ relates to the average number of synaptic contacts which represents the interactions between main cells and interneurons.

This model can be described through this set of nine first-order differential equations which are stated below [12],

$$
\begin{aligned}
& \dot{y}_{0}(t)=y_{5}(t) \\
& \dot{y}_{5}(t)=\operatorname{AaSigm}\left[y_{1}(t)-y_{2}(t)-y_{2}(t)\right] \\
& \dot{y}_{1}(t)=y_{6}(t) \\
& -2 a y_{5}(t)-a^{2} y_{0}(t) \\
& \dot{y}_{6}(t)=A a\left\{p(t)+C_{2} \operatorname{Sigm}\left[C_{1} y_{0}(t)\right]\right\} \\
& -2 a y_{6}(t)-a^{2} y_{1}(t) \\
& \dot{y}_{2}(t)=y_{7}(t) \\
& \dot{y}_{7}(t)=B b C_{4} \operatorname{Sigm}\left[C_{a} y_{0}(t)\right] \\
& \dot{y}_{3}(t)=y_{g}(t) \\
& -2 b y_{7}(t)-b^{2} y_{2}(t) \\
& \dot{y}_{g}(t)=G g C_{7} \operatorname{Sigm}\left[C_{5} y_{0}(t)-C_{6} y_{4}(t)\right] \\
& -2 g y_{g}(t)-g^{2} y_{b}(t) \\
& \dot{y}_{4}(t)=y_{9}(t) \\
& \dot{y}_{9}(t)=B b C_{4} \operatorname{Sigm}\left[C_{2} y_{0}(t)\right] \\
& -2 b y_{9}(t)-b^{2} y_{4}(t)
\end{aligned}
$$

This set of equations can be solved by classical numerical integration methods (Runge-Kutta, example) [15].

\section{Published By:}

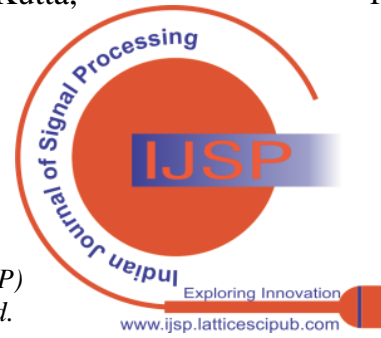


The output of the model, $y(t)=y_{1}(t)-y_{2}(t)-y_{a}(t)$, will emulate the respective EEG signal.

\section{2) Setting the parameter values}

Table- III: Different Parameter for simulating Wendling Model [8]

\begin{tabular}{|c|c|c|}
\hline$\underset{\mathbf{r}}{\text { Paramete }}$ & Interpretation & $\begin{array}{l}\text { Standard } \\
\text { value }\end{array}$ \\
\hline A & Average excitatory synaptic gain & $3.25 \mathrm{mV}$ \\
\hline B & $\begin{array}{l}\text { Average slow inhibitory synaptic } \\
\text { gain }\end{array}$ & $22 \mathrm{mV}$ \\
\hline G & $\begin{array}{l}\text { Average fast inhibitory synaptic } \\
\text { gain }\end{array}$ & $10 \mathrm{mV}$ \\
\hline $\mathrm{a}$ & $\begin{array}{l}\text { The inverse of the Dendritic } \\
\text { average time constant in the } \\
\text { feedback excitatory loop }\end{array}$ & $100 \mathrm{~s}^{-1}$ \\
\hline $\mathrm{b}$ & $\begin{array}{l}\text { The inverse of the Dendritic } \\
\text { average time constant in the } \\
\text { feedback inhibitory loop }\end{array}$ & $50 \mathrm{~s}^{-1}$ \\
\hline g & $\begin{array}{l}\text { The somatic average time constant } \\
\text { in the fast feedback inhibitory loop }\end{array}$ & $500 \mathrm{~s}^{-1}$ \\
\hline $\mathrm{C}_{1}$ & \multirow{2}{*}{$\begin{array}{c}\text { The average number of synaptic } \\
\text { contacts in the excitatory feedback } \\
\text { loop }\end{array}$} & 135 \\
\hline $\mathrm{C}_{2}$ & & $0.8^{*} \mathrm{C}_{1}$ \\
\hline $\mathrm{C}_{3}$ & \multirow{2}{*}{$\begin{array}{l}\text { Average number of synaptic } \\
\text { contacts in the slow feedback } \\
\text { inhibitory loop }\end{array}$} & $0.25 * \mathrm{C}_{1}$ \\
\hline $\mathrm{C}_{4}$ & & $0.25 * \mathrm{C}_{1}$ \\
\hline $\mathrm{C}_{5}$ & \multirow{2}{*}{$\begin{array}{l}\text { Average number of synaptic } \\
\text { contacts in the fast feedback } \\
\text { inhibitory loop }\end{array}$} & $0.3^{*} \mathrm{C}_{1}$ \\
\hline $\mathrm{C}_{6}$ & & $0.1 * \mathrm{C}_{1}$ \\
\hline $\mathrm{C}_{7}$ & $\begin{array}{l}\text { Average number of synaptic } \\
\text { contacts between slow and fast } \\
\text { inhibitory interneurons }\end{array}$ & $0.8^{*} \mathrm{C}_{1}$ \\
\hline$e_{o}, v_{o}, r$ & $\begin{array}{l}\text { Parameters of the nonlinear } \\
\text { asymetric sigmoid function }\end{array}$ & $\begin{array}{l}e_{o}=6 m V \\
v_{o}=2.5 s^{-1} \\
, r=0.5 m V^{-1}\end{array}$ \\
\hline
\end{tabular}

Table- IV: Different Parameter values for simulating six types of seizure-like signals from Wendling Model [12]

\begin{tabular}{|l|l|l|l|l|l|l|}
\hline Signal & \multicolumn{1}{|c|}{$\mathbf{1}$} & \multicolumn{1}{|c|}{$\mathbf{2}$} & \multicolumn{1}{|c|}{$\mathbf{3}$} & \multicolumn{4}{|c|}{$\mathbf{4}$} & \multicolumn{1}{|c|}{$\mathbf{5}$} & \multicolumn{1}{|c|}{} \\
\hline A & 3.25 & 5.6 & 7 & 7 & 7 & 7 \\
\hline B & 22 & 47 & 35 & 15 & 10 & 19 \\
\hline G & 10 & 25 & 10 & 10 & 25 & 3 \\
\hline
\end{tabular}

\section{3) Designing the model}

The subsystem is designed for the linear pulse-to-wave converter operation for the Wendling model is similar to Fig. 4. Here three impulse responses have been used, all this model is similar to Fig. 4 using different parameter values excitatory, slow inhibitory, and fast inhibitory feedback.

The Wendling model has been designed with some subsystems in Simulink. The designed model is shown in Fig. 6.

\section{RESULT ANALYSIS}

If After designing these three models by using the differential equations and the non-linear asymmetric sigmoid function, then by setting all parameters value in different EEG Model, different EEG signals have been generated.

From the Lopes da Silva model, the simulated output signal is like the Alpha Rhythm of brain spontaneous signal as shown in Fig. 7 Which is very close to Alpha rhythms of our brain.

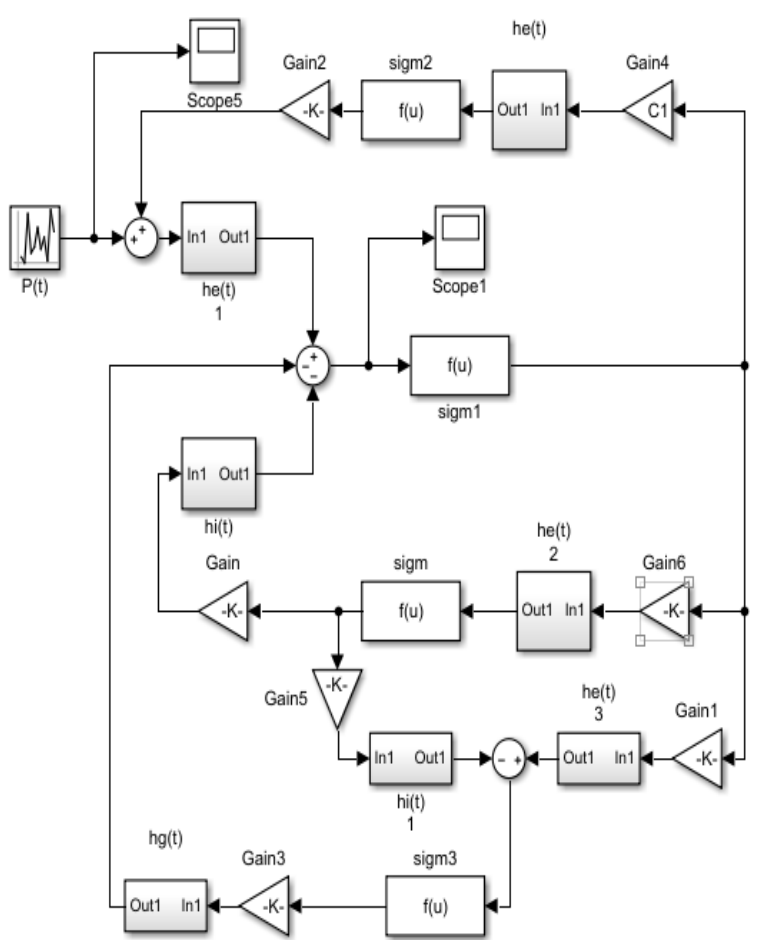

Fig. 6.Figure for designing Wendling model in Simulink.

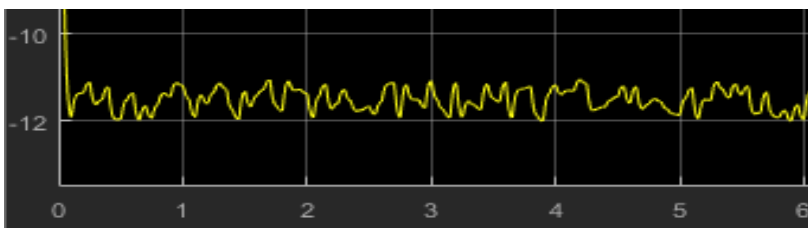

Fig. 7.Simulated alpha wave from Lopes da Silva model.

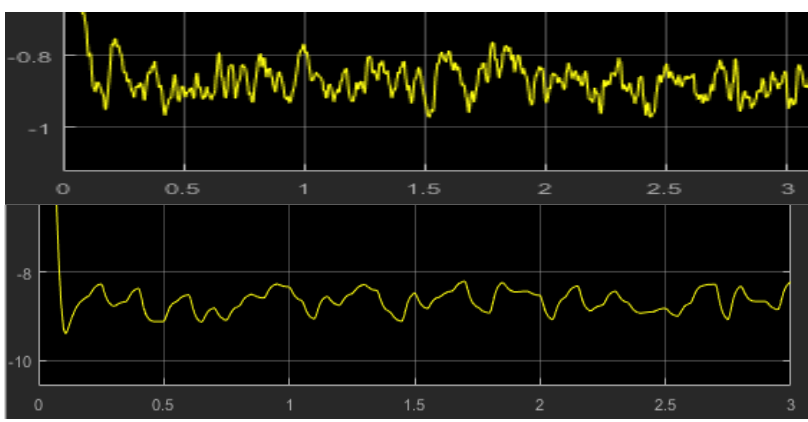

Fig. 8.Simulated Alpha and Beta wave from Jansen-Rit model.

From the Jansen-Rit model, two types of brain waves have been generated which are Alpha rhythm and Beta rhythm. Alpha and Beta signals have been generated by setting all parameters constant and changing the average number of synaptic contacts in the excitatory and inhibitory feedback loop $\left(\mathrm{C}_{1}, \mathrm{C}_{2}, \mathrm{C}_{3}, \mathrm{C}_{4}\right)$ which is very close to our real brain activity as shown in Fig. 8.

\section{Published By:}


To simulate Alpha and Beta rhythms, the values of average number of synaptic contacts, $\mathrm{C}_{1}=135$ and 1350 and corresponding $\mathrm{C}_{2}=0.8 * \mathrm{C}_{1}, \mathrm{C}_{3}=0.25^{*} \mathrm{C}_{1}, \mathrm{C}_{4}=0.25^{*} \mathrm{C}_{1}$ which is shown in Table I.

The Wendling model focuses on high-frequency (gamma band) EEG action, the foremost characteristic electrophysiological pattern in focal seizures of human epilepsy. By setting all parameters, changing the average excitatory, slow inhibitory, and fast inhibitory synaptic gain (A, B, G) as shown in Table IV, and taking all parameters constant six types of seizure-like waves have been generated as shown in Fig. 9

(1)

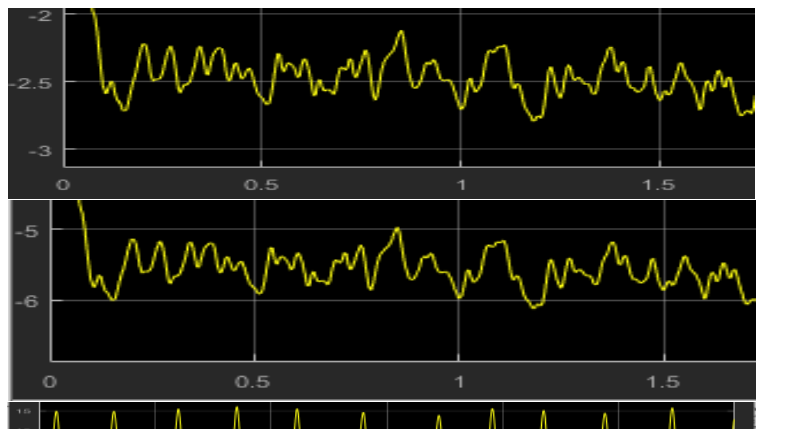

(3)

(4)

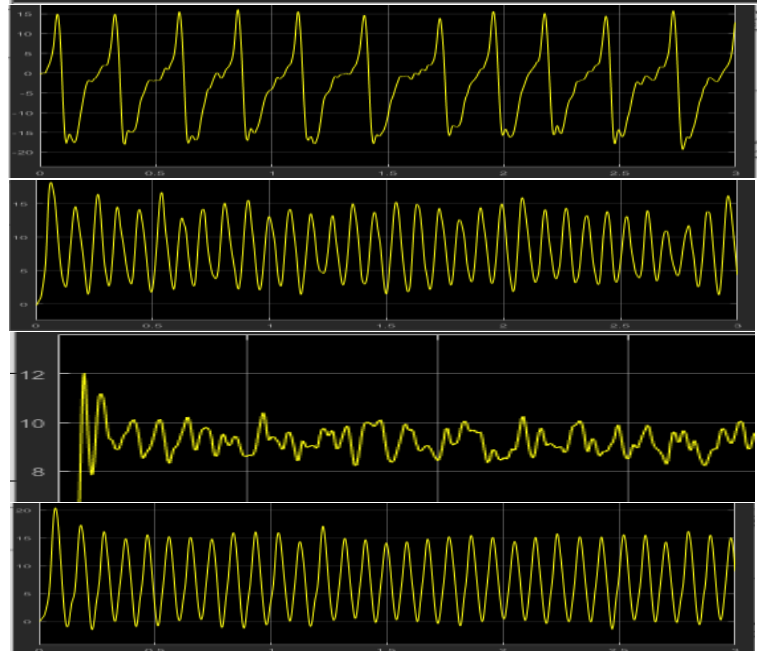

Fig. 9.Simulated six types (Normal background activity, Sporadic Spikes, Sustained discharge of spike, Slow rhythmic activity, Low voltage rapid activity, Slow quasi-sinusoid activity) of the seizure-like signal from Wendling model.

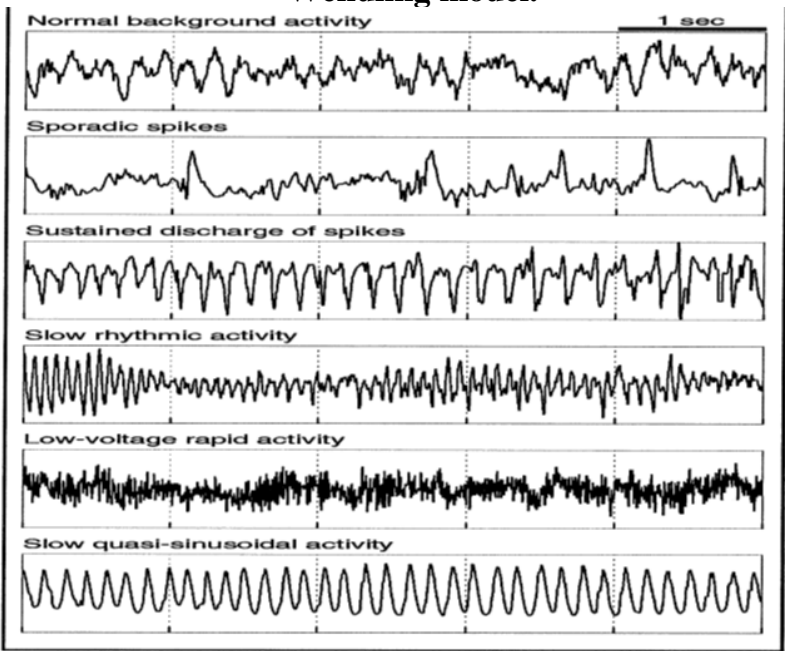

Fig. 10. Real-Depth EEG Signal [8].
From Fig. 10 and Fig.11, it has been shown shat simulated EEG signals from the Wendling model and Real-Depth EEG Signals are very closely related to each other.

\section{CONCLUSION}

The neural mass model enables us to emulate different highly complex EEG rhythms and determine the influence of different parameters on brain activity. The structures of models, analytic descriptions, the important parameters similar to anatomical data, and the relationship among them have been discussed to better understanding and imagination how the model works like the real functionality of the brain. Here the models have been described firstly with analogous to our brain functionality. Then the mathematical model with mathematical equations and many differential equations have been represented to generate EEG-like signal (Alpha and Beta rhythms) from the Lopes da Silva model and Jansen-Rit model, and the seizure-like signal from Wendling model. Then using the differential equations for the linear impulse response and the non-linear sigmoid function have been designed by different simulation blocks of Simulink. Different subsystems have been created to make the model reader-friendly and to reduce ambiguity. The parameter values have been collected from the Matlab workspace. By changing different parameters values different signals have been generated. It has been found that the properties of the simulated signals are very similar to the properties of the real-depth EEG signals

\section{REFERENCES}

1. J. Satheesh Kumar, P. Bhuvaneswari, Analysis of Electroencephalography (EEG) Signals and Its Categorization-A Study, Procedia Engineering, Volume 38, 2012, Pages 2525-2536, ISSN 1877-7058, https://doi.org/10.1016/j.proeng.2012.06.298. [CrossRef]

2. Jansen, B.H., Rit, V.G. Electroencephalogram and visual evoked potential generation in a mathematical model of coupled cortical columns. Biol. Cybern. 73, 357-366 (1995) https://doi.org/10.1007/BF00199471 [CrossRef]

3. Al-Nashash, Hasan et al. "EEG signal modeling using adaptive Markov process amplitude." IEEE transactions on bio-medical engineering vol. 51,5 (2004): 744-51. doi:10.1109/TBME.2004.826602 [CrossRef]

4. F. Shayegh, R. A. Fattahi, S. Sadri, K. Ansari-Asl, "A Brief Survey of Computational Models of Normal and Epileptic EEG Signals: A Guideline to Model-based Seizure Prediction", Journal of Medical Signals and Sensors, vol. 1, no. 1, pp. 62-72, 2011. [CrossRef]

5. Zavaglia, Melissa, Filippo Cona, and Mauro Ursino. "A neural mass model to simulate different rhythms in a cortical region." Computational intelligence and neuroscience 2010 (2010) [CrossRef]

6. Freeman, W J. "Models of the dynamics of neural populations." Electroencephalography and clinical neurophysiology. Supplement ,34 (1978): 9-18.

7. Lopes da Silva, F H et al. "Model of brain rhythmic activity. The alpha-rhythm of the thalamus." Kybernetik vol. 15,1 (1974): 27-37. doi:10.1007/BF00270757. [CrossRef]

8. Wendling, F et al. "Epileptic fast activity can be explained by a model of impaired GABAergic dendritic inhibition." The European journal of $\begin{array}{llll}\text { neuroscience } & \text { vol. } & 15,9 & \text { (2002): }\end{array}$ doi:10.1046/j.1460-9568.2002.01985.x. [CrossRef]

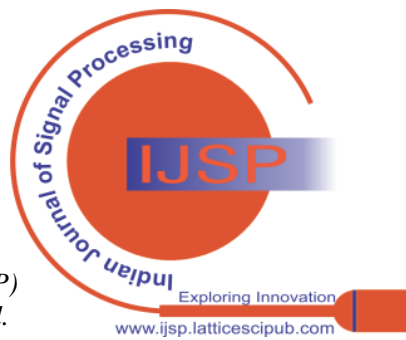


9. Robinson, P A et al. "Prediction of electroencephalographic spectra from neurophysiology." Physical review. E, Statistical, nonlinear, and soft matter physics vol. 63,2 Pt 1 (2001): 021903. doi:10.1103/PhysRevE.63.021903. [CrossRef]

10. David, Olivier, and Karl J. Friston. "A neural mass model for MEG/EEG:: coupling and neuronal dynamics." NeuroImage 20.3 (2003): 1743-1755. [CrossRef]

11. Grimbert, François, and Olivier Faugeras. "Bifurcation analysis of Jansen's neural mass model." Neural computation vol. 18,12 (2006): 3052-68. doi:10.1162/neco.2006.18.12.3052. [CrossRef]

12. S. Geng, W. Zhou, Q. Yuan and Z. Ma, "Bifurcation phenomenon of wendling's EEG model," International Symposium on Bioelectronics and Bioinformations 2011, Suzhou, 2011, pp. 111-114, doi: 10.1109/ISBB.2011.6107658. [CrossRef]

13. E. Dong and Z. Liang, "The multi-frequency EEG rhythms modeling based on two-parameter bifurcation of neural mass model," 2014 IEEE International Conference on Mechatronics and Automation, 2014, pp. 1564-1569, doi: 10.1109/ICMA.2014.6885933. [CrossRef]

14. Tömböl T. Short neurons and their synaptic relations in the specific thalamic nuclei. Brain Res. 1967 Jan 20;3(4):307-26. doi: 10.1016/0006-8993(67)90095-9. PMID: 4166255. [CrossRef]

15. W. H. Press, S. A. Teukolsky, W. T. Vetterling, B. P. Flannery, Numerical Recipes in C: the Art of Scientific Computing, Cambridge: Cambridge University Press, 1992, ch. 16.

\section{AUTHORS PROFILE}

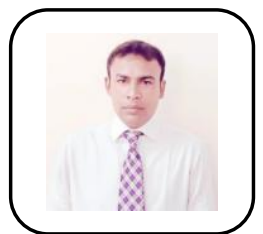

Sheikh Md. Rabiul Islam, received the B.Sc. In Engineering (ECE) Khulna University, Bangladesh in December 2003, and M.Sc. in Telecommunication Engineering from the University of Trento, Italy, in October 2009 and Ph.D. from University of Canberra, Australia, in 2015. He joined as a Lecturer in the department of Electronics and Communication Engineering of Khulna University of Engineering \& Technology, Khulna, in 2004, where he is joined an Assistant Professor in the same department in the effect of 2008.Also he joined Associate Professor in 2015 and joined as Professor in 2018 in the same department. He has given four M.Sc. Engineering at Dept. of ECE and one M.Sc. Engineering (BME) at Dept of Biomedical Engineering. He is also completed four projects by funded university grant commission. Now He has published 2 book chapters, 30 Journal and 33 International conferences. His research interests include biomedical signal \& image processing, VLSI for Biomedical signal and image processing, Machine learning, Embedded system. He is an IEEE member.

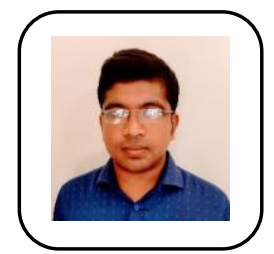

Md. Shakibul Islam, was born in Faridpur, Bangladesh, in 1998. He is currently an undergraduate student in the department of Electronics and Communication Engineering at Khulna University of Engineering \& Technology, Khulna, Bangladesh. His current areas of interest are IoT based medical equipment, EEG-based mental health monitoring, Brain computer interface,

Machine Learning, Deep Learning \& Neural Networks, and Automation.

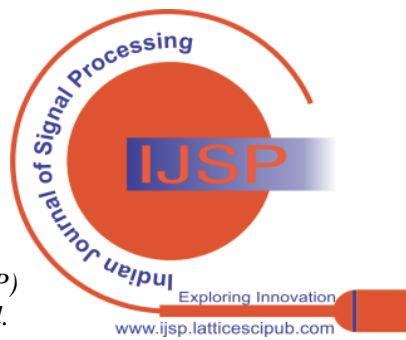

